\title{
Extremal Traces on Some Group-Invariant $C^{*}$-Algebras. $\mathbb{I}^{\prime \prime}$
}

By

\author{
Geoffrey L. PRICE*
}

\begin{abstract}
We consider product-type actions of a compact connected Lie group $G$ on a UHF-algebra $\mathfrak{A}$ of Glimm type $n^{\infty}$. Let $\mathfrak{A}^{G} \subseteq \mathfrak{A}^{T}$ be the fixed-point subalgebras of $\mathfrak{A}$ under the action of $G$ and of a maximal torus $T$ of $G$. We show that the fibre of extremal traces of $\mathfrak{X}^{T}$ extending an extremal trace on $\mathfrak{A}^{G}$ is a single orbit under a natural action of the Weyl group of $G$. We apply this result to classify the extremal traces of $\mathfrak{A}^{G}$.
\end{abstract}

\section{$\S 1$. Introduction}

Let $G$ be a connected compact Lie group, with $T$ a maximal torus of $G$. From a continuous representation $\beta: G \rightarrow \operatorname{Aut}(B)$ of $G$ as *-automorphisms of an $n \times n$ matrix algebra $B$, one may form a strongly continuous representation $\alpha$ of $G$ of product type on the UHF algebra $\mathfrak{A}=\bigotimes_{k \geqslant 1} B$ of Glimm type $n^{\infty}$. We consider the $C^{*}$-subalgebra $\mathfrak{H}^{G}$ of elements fixed by $\alpha$; and by restriction of $\alpha$ to $T$, the corresponding fixed point subalgebra $\mathfrak{A}^{T}$.

In [7] it was shown that any extremal trace on $\mathfrak{P I}^{G}$ arises as the restriction of a symmetric product state $\otimes \omega$ on $2 \mathfrak{l}$. Using some results from [7] as well as some $K_{0}$-theoretic techniques, Handelman [4] has recently shown that any extremal trace on $\mathfrak{Q}^{G}$ admits an extension to an extremal trace on $\mathfrak{A}^{T}$. In this paper we show that, for a fixed extremal trace $\tau$ on $\mathfrak{P}^{G}$, the fibre of extremal traces of $\mathfrak{Q I}^{T}$ which restrict to $\tau$ is a single orbit under the action of the Weyl group of $G$. Since it is relatively straightforward to describe the space of tracial states of $\mathfrak{Y}^{T}$ (see [4], [10], and below), a combination of Handelman's extendability results with the result above yields a complete characterization of the

Communicated by H. Araki, August 25, 1984.

* Department of Mathematics, United States Naval Academy, Annapolis, MD 21402, U.S. A.

1) Supported in part by NSF Grant No. MCS-8202290. 
extremal traces of $\mathfrak{Q P}^{G}$.

\section{§2. Fixed Point Algebras of Product Actions}

Throughout this paper $\mathfrak{I}$ shall denote a uniformly hyperfinite $C^{*}$-algebra of type $n^{\alpha}$. We write $\mathfrak{V}$ as the infinite tensor product $\mathfrak{N}=\otimes_{k \in N} B_{k}$, where each $B_{k}$ is a copy of an $n \times n$ matrix algebra $B$ over $C$. For $m \in N \in N$, let $\mathfrak{N}_{m}=\bigotimes_{k=1}^{m} B_{k}$. Then $\mathfrak{U}_{m}$ consists of finite sums of elements of the form $x_{1} \otimes \cdots \otimes x_{m}, x_{k} \in B_{k}$. Moreover, we may view $\mathfrak{A}_{m}$ as a subalgebra of $\mathscr{\mathfrak { C }}$ by identifying it with the canonical embedding of $\bigotimes_{k=1}^{m} B_{k}$ into $\mathfrak{N}$. Under this identification $\mathfrak{A}_{m}$ and $\mathfrak{N}$ have a common identity, $\mathbf{1}$.

We describe matrix units for $\mathfrak{A l}_{m}$ as follows. First let $\left\{e_{i j}: 1 \leqslant i, j \leqslant n\right\}$ be matrix units for $B$; i.e., $e_{i j} e_{p q}=\delta_{j p} e_{i q}$, and the diagonal elements have sum $\sum_{i=1}^{n} e_{i i}$ $=1$. Denote by $\left\{e_{i j}^{k}: 1 \leqslant i, j \leqslant n\right\}$ the corresponding matrix units for $B_{k}$. If $\mathfrak{I}_{m}$ is the set of ordered $m$-tuples $I=\left(i_{1}, i_{2}, \ldots, i_{m}\right), 1 \leqslant i_{k} \leqslant n$, then the elements $E_{I J}$ $=e_{i_{1} j_{1}}^{1} \otimes \cdots \otimes e_{i_{m} j_{m}}^{m}$ are matrix units for $\mathfrak{A}_{m}$. In particular $E_{I J} E_{K L}=\delta_{J K} E_{I L}, I, J$, $K, L \in \mathfrak{I}_{m}$, and the diagonal elements $E_{I I}$ have sum equal to the identity in $\mathfrak{A}_{m}$.

Let $G$ be a compact connected Lie group, with maximal torus $T$. Let $\beta: G \rightarrow \operatorname{Aut}(B)$ be a faithful continuous representation of $G$, where we assume that we have chosen matrix units for $B$ so that $\left.\beta\right|_{T}$ embeds diagonally, i.e., for $t \in T, \beta_{t}=\operatorname{Ad}\left(V_{t}\right)$, where $V_{t}$ is the diagonal unitary

$$
V_{t}=\sum_{i=1}^{n}\left(\gamma_{i}, t\right) e_{i i}
$$

We then construct the corresponding strongly continuous representation $\alpha$ of product automorphisms of $\mathfrak{A}$, where $\alpha_{g}=\otimes_{k \in \boldsymbol{N}} \beta_{g}$ for $g \in G$.

We denote by $\mathfrak{I}^{G}$ (respectively, $\mathfrak{I T}^{T}$ ) the $C^{*}$-subalgebra of $\mathfrak{Q}$ of fixed points under the action of $\alpha$ (respectively, of $\left.\alpha\right|_{T}$ ). Clearly $\mathfrak{Q l}^{G} \subseteq \mathfrak{Q l}^{T} \subseteq \mathfrak{Q}$. Moreover, if $\mu$ is the Haar probability measure on $G$, it is easy to check that $\mathfrak{P l}^{G}$ coincides with the image of the conditional expectation $\Phi$, given by

$$
\Phi(x)=\int_{G} \alpha_{g}(x) d \mu(g), \quad x \in \mathfrak{U} .
$$

$\mathfrak{P}^{G}$ is an AF-algebra: in fact [7] $\mathfrak{H}^{G}$ is the uniform closure of the ascending union of algebras $\mathfrak{Q I}_{m}^{G}=\mathfrak{U}_{m} \cap \mathfrak{U}^{\mathbf{G}}, m \in \boldsymbol{N}$.

We recall from [7] that there exists an embedding of $S(\infty)$, the discrete group of finite permutations on the symbols of $N$, into the unitary group $\mathfrak{I}(\mathfrak{A})$ 
of $\mathfrak{Y}$. Let $\sigma \rightarrow U_{\sigma}$ denote this embedding: then we have, for example,

$$
U_{\sigma}\left(e_{i j}^{k}\right)\left(U_{\sigma}\right)^{*}=e_{i j}^{\sigma(k)} .
$$

From [7], $S(\infty) \subseteq \mathfrak{Q}^{G}$. Hence, if $\mathbb{C}$ denotes one of the algebras $\mathfrak{Q T}, \mathfrak{P l}^{G}, \mathfrak{V}^{T}$, we shall say that a state $\omega$ on $\mathbb{C}$ is symmetric [9] if $\omega=\omega \circ \mathrm{Ad}\left(U_{\sigma}\right)$, all $\sigma \in S(\infty)$. In particular, a tracial state on $\mathbb{C}$ must be symmetric. Observe also that a product state $\omega=\otimes_{k \in N} \omega_{k}$ on $\mathfrak{T}$ is symmetric if and only if all of the states $\omega_{k}$ coincide. By abuse of notation we write symmetric product states in the form $\omega=\otimes \omega_{P}$, where $P \in B_{1}(=B)$ is the density matrix satisfying $\omega_{1}(x)=\operatorname{Tr}_{B_{1}}(P x)$, all $x \in B_{1}$.

With the following two results we obtain a characterization of the extremal traces on $\mathfrak{S}^{T}$. Although this characterization is already implicit in both [5] and [10], we provide an independent proof arising from a somewhat different point of view than in these papers. Moreover, our intermediate step, Proposition 2.1, is needed in the form given to sharpen the transitivity result in Theorem 3.1.

Proposition 2.1. Let $\tau$ be an extremal trace of $\mathfrak{I}^{T}$. Then $\tau$ is the restriction of a symmetric product state $\omega=\otimes \omega_{D}$, where $D \in B$ is a diagonal density matrix.

Proof. First observe that for $k \in \mathbb{N}, 1 \leqslant i \leqslant n$, the diagonal elements $e_{i i}^{k}$ lie in $\mathfrak{U}^{T}$, from Equation (1). Hence for fixed $m$, the diagonal elements $\mathbb{E}_{I I} \in \mathfrak{Q}_{m}$, $I \in \mathfrak{I}_{m}$, lie in $\mathfrak{P}_{m}^{T}$. Now for any $x \in \mathfrak{P}_{m}^{T}$, write

$$
x=\sum_{1, J \in \Re m} a_{I J} E_{I J}, \quad a_{I J} \in \mathbb{C} .
$$

Since $\tau$ is a trace, we have

$$
\begin{aligned}
& \tau(x)=\tau(\mathbb{1} x)=\tau\left(\left[\sum_{I \in \Im^{\prime} m} E_{I I}\right] x\right)=\tau\left(\sum_{I \in \Upsilon_{m}}\left(E_{I I} x E_{I I}\right)\right)
\end{aligned}
$$

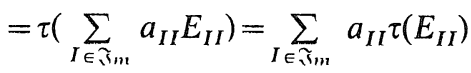

Fix $E_{I I}=e_{i_{1} i_{1}}^{1} \otimes \cdots \otimes e_{i_{m} i_{m}}^{m}$ in $\mathfrak{U}_{m}^{T}$. Since $\tau$ is extremal it is multiplicative, by $[7$, Theorem 3.2], so that

$$
\tau\left(E_{I I}\right)=\prod_{k=1}^{m} \tau\left(e_{i_{k} i_{k}}^{k}\right)
$$

Since $\tau$ is symmetric we have

$$
\tau\left(E_{I I}\right)=\prod_{k=1}^{m} \tau\left(e_{i_{k} i_{k}}^{1}\right)
$$

Now let $D \in B_{1}$ be the diagonal density matrix $D=\sum_{i=1}^{n} \tau\left(e_{i i}^{1}\right) e_{i i}^{1}$, and let $\omega=\otimes \omega_{D}$ 
be the corresponding product state. Then a straightforward computation shows that $\omega(x)=\tau(x), x \in \mathfrak{A}_{m}^{T}$, all $m \in N$. Therefore $\left.\omega\right|_{\mathfrak{A}^{T}}$ and $\tau$ agree on $\underset{m \in N}{\bigcup} \mathfrak{A}_{m}^{T}$, so that $\tau=\left.\omega\right|_{\mathfrak{T} T}$, by continuity.

We next determine which diagonal $D$ yield traces on $\mathfrak{A}^{T}$ as in the proposition. To do so we first observe some facts about the Wedderburn decomposition of $\mathfrak{U}_{m}^{T}$ into sums of matrix algebras. Let $E_{I I}\left(I=\left(i_{1}, i_{2}, \ldots, i_{m}\right) \in \mathfrak{I}_{m}\right)$ be a minimal diagonal matrix element of $\mathfrak{U}_{m}^{T}$, and define a representation $\gamma_{I}$ of $T$ by

$$
\gamma_{I}=\prod_{k=1}^{m} \gamma_{i_{k}}
$$

The relation $\left(\gamma_{I}, t\right) E_{I I}=\left(\bigotimes_{k=1}^{m} V_{t}\right) E_{I I}, t \in T$, follows easily from Eq. (1). Now suppose $I, J \in \mathfrak{I}_{m}$ and that $E_{I I}, E_{J J}$ lie in the same direct summand of $\mathfrak{U}_{m}^{T}$. Then $E_{I I}=U E_{J J}\left(U^{*}\right)$, for some $U \in \mathfrak{A}_{m}^{T}$, so that

$$
\left(\gamma_{I}, t\right) E_{I I}=\left(\bigotimes_{k=1}^{m} V_{t}\right) E_{I I}=U\left[\left(\bigotimes_{k=1}^{m} V_{t}\right) E_{J J}\right]\left(U^{*}\right)=\left(\gamma_{J}, t\right) E_{I I}, \quad t \in T,
$$

i.e., $\gamma_{I}=\gamma_{J}$. Conversely, if $\gamma_{I}=\gamma_{J}$ for $I, J \in \mathfrak{I}_{m}$, then note that

$$
\alpha_{t}\left(E_{I J}\right)=\operatorname{Ad}\left(\bigotimes_{k=1}^{m} V_{t}\right)\left(E_{I J}\right)=\left(\gamma_{1}, t\right) E_{I J}\left(\overline{\gamma_{J}, t}\right)=E_{I J},
$$

so that $E_{I J} \in \mathfrak{A O}_{m}^{T}$. Let $U \in \mathfrak{P}_{m}^{T}$ be the unitary operator $\left[1+\left(E_{I J}+E_{J I}\right)\right.$ $\left.-\left(E_{I I}+E_{J J}\right)\right]$. Then $E_{I I}=U E_{J J}\left(U^{*}\right)$, hence $E_{I I}$ and $E_{J J}$ are in the same direct summand of $\mathfrak{A}_{m}^{T}$ (cf. [2, Lemma 5.2]).

Now suppose $D=\sum_{i=1}^{n} \lambda_{i} e_{i i}$ is a diagonal density matrix of $B$, and $\tau_{D}$ $=\left.\otimes \omega_{D}\right|_{\mathscr{Y} T} . \quad$ If $\tau_{D}$ is a trace then $\tau_{D}\left(E_{I I}\right)=\tau_{D}\left(E_{J J}\right)$ for $E_{I I}, E_{J J}$ in the same summand of $\mathfrak{U}_{m}^{T}$. Computing this gives $\prod_{k=1}^{m} \lambda_{i_{k}}=\prod_{k=1}^{m} \lambda_{j_{k}}\left(\right.$ where $\left.\left(j_{1}, \ldots, j_{m}\right)=J\right)$ ) Hence if $\gamma_{I}=\gamma_{J}$ then $\lambda_{I}=\lambda_{J}, I, J \in \mathfrak{I}_{m}$, where $\lambda_{I}=\prod_{k=1}^{m} \lambda_{i_{k}}$. Conversely, if $\lambda_{I}=\lambda_{J}$ for all $I, J \in \mathfrak{A}_{m}$ such that $\gamma_{I}=\gamma_{J}$, then one shows easily that $\left.\tau_{D}\right|_{\mathscr{R}_{m}^{T}}$ is a trace, $m \in N$, so that $\tau_{D}$ is a trace on $\mathfrak{A}^{T}$ by continuity. Hence we have the following.

Theorem 2.2. Let $\mathfrak{A}^{T}$ be the fixed point algebra of $\mathfrak{A}$ under the product action $\alpha_{t}=\operatorname{Ad}\left(\otimes V_{t}\right)$ of the torus $T$, where $V_{t}, t \in T$ is given by (1). Then there is a one to one correspondence, given by $\tau=\left.\otimes \omega_{D}\right|_{\mathscr{I}_{T}}$, between the extremal traces $\tau$ of $\mathfrak{Q}^{T}$ and diagonal density matrices $D=\sum_{i=1}^{n} \lambda_{i} e_{i i}$ of $B$ which satisfy

$$
\prod_{k=1}^{m} \lambda_{i_{k}}=\prod_{k=1}^{m} \lambda_{j_{k}}
$$

whenever 


$$
\prod_{k=1}^{m} \gamma_{i_{k}}=\prod_{k=1}^{m} \gamma_{j_{k}}
$$

$I, J \in \mathfrak{J}_{m}$, all $m \in N$.

Proof. All that remains to be observed is that the trace $\tau=\left.\otimes \omega_{D}\right|_{\mathscr{I}^{T}}$ is extremal for $D$ satisfying (*). But this follows immediately since $\tau$ is the restriction of a product state, [10, Theorem 4.4].

Example. Let $\mathfrak{A}=\bigotimes_{k \geqslant 1} B$ be the UHF algebra of type $3^{\infty}$. Consider the representation in $B$ of $G=S O(3)$ so that $G$ contains the group $T=\left\{e^{i \theta} e_{11}+\right.$ $\left.e^{-i \theta} e_{22}+e_{33}: \theta \in[0,2 \pi)\right\}$ as a maximal torus. Using the notation of Theorem 2.2 , the product state $\otimes \omega_{D}$ restricts to a trace on $\mathfrak{I}^{T}$ if and only if $\lambda_{1} \lambda_{2}=\left(\lambda_{3}\right)^{2}$.

\section{§3. The Orbit of Extensions}

Let $\tau$ be an extremal trace of $\mathfrak{I}^{G}$. By [4, Theorem 1.3] there exists an extremal trace $\tau_{0}$ on $\mathfrak{Q}^{T}$ extending $\tau$. It follows from [1] that any other such extension lies in the $G$-orbit of $\tau_{0}$. We prove a sharpened version of this below.

If $T$ is a maximal torus of $G$, we denote by $N(T)$ the normalizer group of $T$, i.e., $N(T)=\left\{g \in G: g T\left(g^{-1}\right)=T\right\}$, and by $W$ the Weyl group $N(T) / T$. The following result is an analogue of (and has a proof virtually identical to) the classical result that two elements of $T$ are conjugate in $G$ if and only if they are conjugate in $W$, (see [11, Lemma 4.33]).

Theorem 3.1. Let $\tau$ be an extremal trace on $\mathfrak{H}^{G}$. Then $W$ acts transitively on the fibre of extremal traces of $\mathfrak{Q}^{T}$ extending $\tau$.

Proof. Note that for $k \in N(T), \alpha_{k}: \mathfrak{A}^{T} \rightarrow \mathfrak{A I}^{T}$, so that we may define automorphisms $\alpha_{w}$ of $\mathfrak{U}^{T}, w \in W$, by $\alpha_{w}(x)=\alpha_{k}(x), x \in \mathfrak{U}^{T}$, where $w=k T$. Hence if $\tau_{0}$ is an extremal trace of $\mathfrak{Q}^{T}$ extending $\tau$, so is $\tau_{0} \circ \alpha_{w}$.

Now suppose that $\tau_{0}, \tau_{1}$, are extremal traces of $\mathfrak{Q}^{T}$, extending $\tau$, and let $D_{i} \in B, i=0,1$, be diagonal density matrices such that $\tau_{i}=\left.\left(\otimes \omega_{D_{i}}\right)\right|_{\mathscr{A}_{T}}$. By $[1$, Theorem II.1 (1)] (see also [3, Theorem 5.4.24]) there is a $g \in G$ such that $\tau_{0}=\tau_{1} \circ \alpha_{g}$. From this it follows easily that $D_{0}=\beta_{g}\left(D_{1}\right)$. Let $N=N\left(D_{1}\right)=$ $\left\{k \in G: \beta_{k}\left(D_{1}\right)=D_{1}\right\}$. Then $T \subseteq N$, since $D_{1}$ is diagonal. Also $g^{-1} T g \subseteq N$, for if $t \in T$,

$$
\beta_{g^{-1} t g}\left(D_{1}\right)=\beta_{g^{-1} t}\left[\beta_{g}\left(D_{1}\right)\right]=\beta_{g^{-1}}\left[\beta_{t}\left(D_{0}\right)\right]=\beta_{g^{-1}}\left(D_{0}\right)=D_{1} .
$$

Hence $T, g^{-1} \mathrm{Tg}$ are maximal tori of $N$, so there exists [11, Corollary 4.23] $h \in N$ 
with $h^{-1}\left(g^{-1} T g\right) h=T$. Set $n=g h(\in N(T))$. Then $\beta_{n}\left(D_{1}\right)=D_{0}$, so that $\tau_{1}=$ $\tau_{0} \circ \alpha_{n}$, as in the proof of the theorem.

Remark. From Handelman's result on extendability and the result above, we have $\mathfrak{E}\left(\mathfrak{H}^{G}\right)=\left(\mathfrak{E}\left(\mathfrak{H}^{T}\right)\right)^{W}$, where $\mathfrak{E}(\mathfrak{C})$ denotes the extremal traces of a $C^{*}$. algebra $\mathfrak{C}$. We refer the reader to [5] for some illuminating examples of the tracial state spaces of $\mathfrak{U}^{\mathbb{G}}$ and of $\mathfrak{U}^{T}$.

\section{Acknowledgements}

We are grateful to David Handelman for correspondence, and to Jerry Kaminker and Neal Rothman for helpful conversations. We thank also the referee for bringing the paper of Araki, Haag, Kastler, and Takesaki to our attention, thereby shortening our proof of Theorem 3.1.

\section{References}

[1] H. Araki, R. Haag, D. Kastler, and M. Takesaki, Extension of KMS states and chemical potential, Comm. Math. Phys. 53, (1977), 97-134.

[2] O. Bratteli, Inductive limits of finite-dimensional $C^{*}$-algebras, Trans. Amer. Math. Soc. 171, (1972), 195-234.

[3] O. Bratteli and D. W. Robinson, Operator algebras and quantum statistical mechanics, I, II, Springer-Verlag, New York, 1979.

[4] D. Handelman, Extendibility of traces on compact group fixed point algebras, eventual positivity of differences of characters, and finite equivalence of matrices of characters, University of Ottawa preprint, 1984.

[5] — - Ordered rings arising from product type actions of connected compact groups, University of Ottawa preprint, 1982.

[6] R. T. Powers. Representations of uniformly hyperfinite algebras and their associated von Neumann rings, Annals of Math. 86, (1967), 138-171.

[7] G. Price, Extremal traces on some group-invariant $C^{*}$-algebras, J. Func. Anal. 49, (1982), 145-151.

[8] E. Størmer, Large groups of automorphisms of $C^{*}$-algebras, Comm. Math. Phys. 5, (1967), 1-22.

[9] - Symmetric states of infinite tensor products of $C^{*}$-algebras, J. Func. Anal. 3, (1969), 48-68.

[10] A. Wassermann, Automorphic actions of compact groups on operator algebras, Thesis University of Pennsylvania, 1981.

[11] J. F. Adams, Lectures on Lie groups, W. A. Benjamin, New York, 1969. 